\title{
The Blazhko Effect in RR Lyrae
}

\author{
Terry J. Teays \\ Computer Sciences Corporation/IUE Observatory
}

\begin{abstract}
The cause of the Blazhko effect, the long-term modulation of the light and radial velocity curves of some RR Lyr stars, is still not understood. The observational characteristics of the Blazhko effect are discussed in $\S 1$. Some preliminary results are presented from aomw recent of campaigns to observe RR Lyr, using the International Ultraviolet Explorer along with ground-based spectroscopy and photometry, throughout a pulsation cycle, at a variety of Blazhko phases. A set of ultraviolet light curves have been generated from low dispersion IUE spectra. In addition, the (visual) light curves from IUE's Fine Error Sensor are analyzed using the Fourier decomposition technique. The values of the parameters $\phi_{21}$ and $R_{21}$ at different Blazhko phases of RR Lyr span the range of values found for non-Blazhko variables of similar period.
\end{abstract}

\section{Characteristics of the Blazhko Effect}

The first allusion to the Blazhko effect was in a paper which included some observations of RW Draconis (Blazhko 1907) published 85 years ago, so the phenomenon has been around for a long time, and yet we still do not have an adequate understanding of it. In brief, the effect is a long term modulation of the amplitude of the light and velocity curves of an RR Lyr star. Some additional details are given below, with special focus on the properties of RR Lyr itself (the brightest RR Lyr star to show the Blazhko effect). The amplitude variation in visual light is typically quite noticeable, for example, in RR Lyr there may be a difference as large as 0.3 magnitude (out of approximately one magnitude pulsational variation), between the extremes of the Blazhko cycle. In addition the shape of the light curve varies as a function of the Blazhko cycle. [A complete set of light curves can be seen in Walraven (1949).] In RR Lyr a "shoulder" appears during rising light, just prior to maximum, in the smaller amplitude Blazhko phases, but which is not present in the larger amplitude phases. Except for this particular feature, the radial velocity curve exhibits changes which are mirror images of those seen in the light curve. In addition, emission is seen in the hydrogen lines during some Blazhko phases, but not others.

Blazhko periods $\left(P_{B}\right)$ generally run from about $20-100^{d}$, i.e. $\approx 100$ times the fundamental pulsation period $(\Pi)$. There does not appear to be any correlation between $\Pi$ and $P_{B}$ (Szeidl 1988). If one compares the amplitude of pulsation for a star showing Blazhko effect to ones with a similar period that does not show it, the 
Blazhko variable's amplitude at maximum most closely resembles the non-Blazhko stars (Szeidl 1988).

The amplitude of the Blazhko effect itself is also modulated on an even longer time scale in a number of RR Lyr stars. RR Lyr itself shows a tertiary period of about 3.8-4.8 years. The Blazhko effect was especially weak during 1963, 1967, 1971, and 1975. For instance, the extensive photometric and spectroscopic study of rising light performed by Preston, Smak, and Paczyński (1965) showed that the Blazhko effect was virtually non-existent in RR Lyr in 1963. Spectroscopically, the variation in the hydrogen emission strength, however, was still observable during this time.

Estimates of the percentage of RR Lyr stars which show the Blazhko effect varies in the range of $15-35 \%$. Given that the effect can all but disappear in RR Lyr at times, as discussed above, one must view these estimates with some caution. Several Bailey Type c variables (overtone pulsators) have been proposed as stars showing Blazhko effect, but it is much harder to study in these lower amplitude pulsators with sinusoidal light curves. In general the Blazhko effect is confined to the RR Lyr stars with shorter period; it is not seen in stars with periods longer than about $0 .{ }^{d} 6$.

\section{Proposed Explanations of the Blazhko Effect}

It is the characteristics that I have listed in $\S 1$ that must be explained by any theory of the cause of the Blazhko phenomenon. There is space here to only summarize the two broad categories of working hypotheses, viz., the magnetic pulsator and various resonance interactions. The former is similar to the oblique magnetic pulsator models which have been used to explain rapidly oscillating Ap stars. In theses theories $P_{B}$ is just the rotation period of the star. Support for a magnetic field modulated by the pulsation and Blazhko periods has been found in the polarization measurements of Romanov, Udovichenko, and Frolov (1987). Unfortunately, the original data were not published, and these results are in need of confirmation. I would strongly urge that observers attempt magnetic field measurements of RR Lyr at a variety of pulsation and Blazhko phases. The second general class of theories proposed are those which invoke resonance interactions with other modes. Examples of these scenarios can be found in Borkowski (1980), Moskalik (1986), and Cox (this conference). None of the proposed explanations mentioned above have met with universal acceptance, due largely to their failure to account for all of the observed phenomenon.

\section{The Multi-wavelength Observing Campaigns}

In order to provide a fairly extensive collection of observations of the Blazhko effect I organized two multi-site, multi-wavelength campaigns during the summers of 1990 and 1991. These campaigns included ground-based photometry and spectroscopy, which was obtained to coincide with observations conducted with the International Ultraviolet Explorer (IUE). The IUE observations covered a complete pulsation cycle, and were repeated at a variety of Blazhko phases. The participants in this project 
are:

\begin{tabular}{|ll|ll}
\hline T. J. Teays & (CSC-IUE) & J. T. Bonnell & (CSC-IUE) \\
T. G. Barnes III & (Texas) & J. M. Nemec & (WSU) \\
E. F. Milone & (Calgary) & E. F. Guinan & (Villanova) \\
E. G. Schmidt & (Nebraska/NSF) & J. Heath & (Texas/Cal' \\
E. Poreti & (Milano-Merate Observatory) & D. G. Schleicher & (Lowell) \\
E. Dutchover & (Texas) & M. Frueh & (Texas) \\
D. Greenlaw & (Texas) & K. Venn & (Texas) \\
\hline
\end{tabular}

We are presently analyzing the large amount of data that were obtained during these two summers, but some preliminary results will be presented below.

\section{a. Photometry}

Ground-based photometry was obtained on a number of nights, and included (at different sites) UBVRHJK bandpasses. This photometry is being used to supplement the photometry provided by IUE's Fine Error Sensor (FES). The FES is an image dissector with an S-20 photocathode (effective wavlength $=5200 \AA$ ) which is used for target identification and tracking. It can serve as a good photometer under controlled conditions, except for the shortcoming that it provides no color information. We have used the FES to collect data during the times between taking spectra, when the spectral camera was being prepared for the next image. From these data we have constructed extensive light curves, which span a complete pulsation cycle (13.6 hours). The FES measurements have been converted into $\mathrm{V}$ magnitudes using the standard IUE calibration (Pérez et al. 1991). The one difficulty was in applying the correction for the (changing) color of the star. For the purposes of this paper we have made use of mean colors, calculated as a function of pulsation phase, from a model of RR Lyr by Bonnell. Figure 1 shows the FES light curves for RR Lyr at two different Blazhko phases, representing the extremes of amplitude that were observed. The Blazhko effect was clearly quite strong during the 1990 campaign!

\section{b. IUE Observations}

The 1990 campaign made use of IUE's long wavelength prime (LWP) camera in its low resolution mode, which has a wavlength range of $\approx 1910-3300 \AA$. A contiguous pair of IUE shifts was used (i.e. 16 hours) in order to completely cover one pulsation cycle. Five such runs were conducted, in order to sample the Blazhko cycle in detail. The time required to read down an image on the LWP camera, and prepare the camera for the next exposure is 28 minutes. In order to increase the time resolution between subsequent spectra, we placed two spectra on the same IUE image, by offsetting them in the aperture. This technique requires additional effort beyond the usual procedures to extract the fluxes properly, but is necessary, to delineate the shape of 

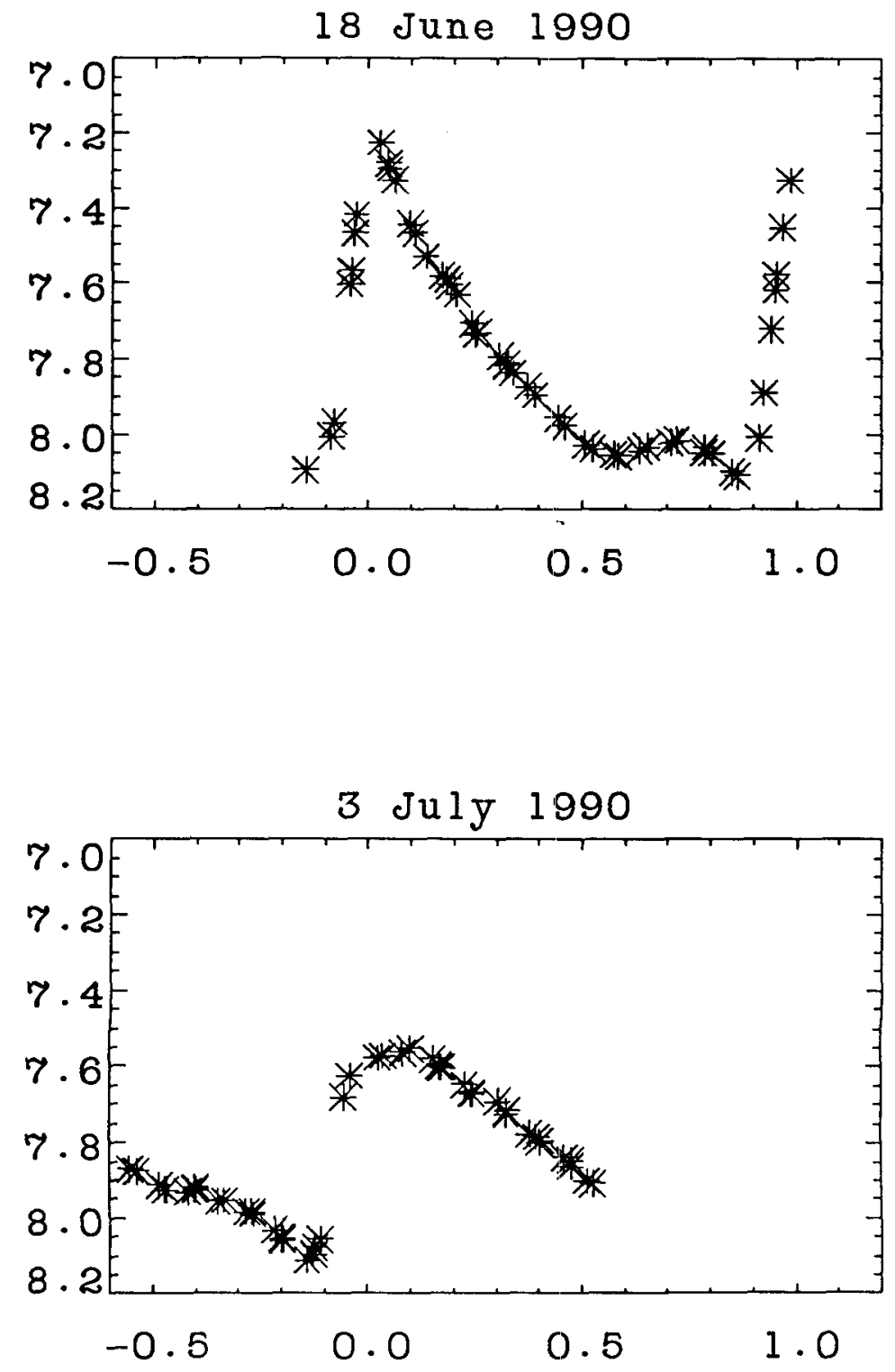

Figure 1. V light curves of RR Lyr at two different Blazhko phases, as measured by IUE's FES. These were selected to show the extremes of the variation in the light curve during the 1990 season. 
the ultraviolet light curve, especially in the rapidly varying, rising light phases. From these spectra we have generated ultraviolet light curves at four wavelengths $(2450$, $2600,2800, \& 3000 \AA$ ) by simply binning the data. These data, when combined with the FES measurements, give the amplitude of the pulsation as a function of wavelength, from the visual through the ultraviolet, which can be directly compared to model predictions.

In 1991 we concentrated on using IUE's short wavelength prime (SWP) camera to obtain low dispersion spectra in the 1150-1975 $\AA$ range. The longer exposure times required in the SWP camera precluded us from obtaining the detailed ultraviolet light curves that we could get with the LWP camera, as well as from getting FES light curves with the necessary time resolution. Rather we concentrated on always getting a spectrum at both minimum and maximum light, so that the ultraviolet amplitude was well determined. We used four IUE shifts of eight hours duration to obtain these spectra, one of which was only two days later than a final pair of shifts that were used for obtaining detailed LWP and FES light curves, as in the 1990 campaign. This allowed us to have one time when we had complete waveleng coverage.

\section{c. Ground-based Spectroscopy}

During these campaigns spectrsocopy was obtained at McDonald, Dominion Astrophysical, and Palomar Observatories. These efforts, though hampered by the usual weather, instrument, and scheduling difficulties were successful on a number of nights. Reduction of these data is not complete, and we still have a great deal of work ahead of us to analyze the results, so in this review I will only mention the nature of the spectra taken $(\sim 100)$. Observations were concentrated on the region of the $\mathrm{H} \gamma$ line or on the $\mathrm{Ca} \mathrm{II} \mathrm{H}$ and $\mathrm{K}$ lines. The former also include some metallic lines, which we can yield some radial velocity information. During the course of the pulsation cycle, one can see variable emission in the line core of $\mathrm{H} \gamma$.

\section{Preliminary Results}

The ultraviolet light curves obtained from the LWP camera have sufficient quality and phase coverage that we have been able to apply the standard Fourier decomposition techniques first introduced in Simon \& Lee (1981). In addition, we have Fourier decomposed the $\mathrm{V}$ light curves obtained from the FES. In Figure 2 we plot the Fourier parameter $\phi_{21}$ vs. $\Pi$ for RR Lyr at the different Blahzko phases which we sampled, and compare it to the sample of field RR Lyr stars used by Simon and Teays (1982). Similarly, Figure 3 shows the Simon \& Lee parameter $R_{21}$ plotted against period. What is seen in both cases is that the values for RR Lyr fall closely within the range of values for the non-Blazhko stars with similar pulsation period. In fact, as RR Lyr goes through its Blazhko cycle its light curve recapitulates the appearance of all field RR Lyr stars of similar period. We have also examined a period vs. amplitude diagram using the FES data, and confirm the results mentioned in Szeidl (1988), namely, that the closest match between Blazhko and non-Blazhko stars occurs 


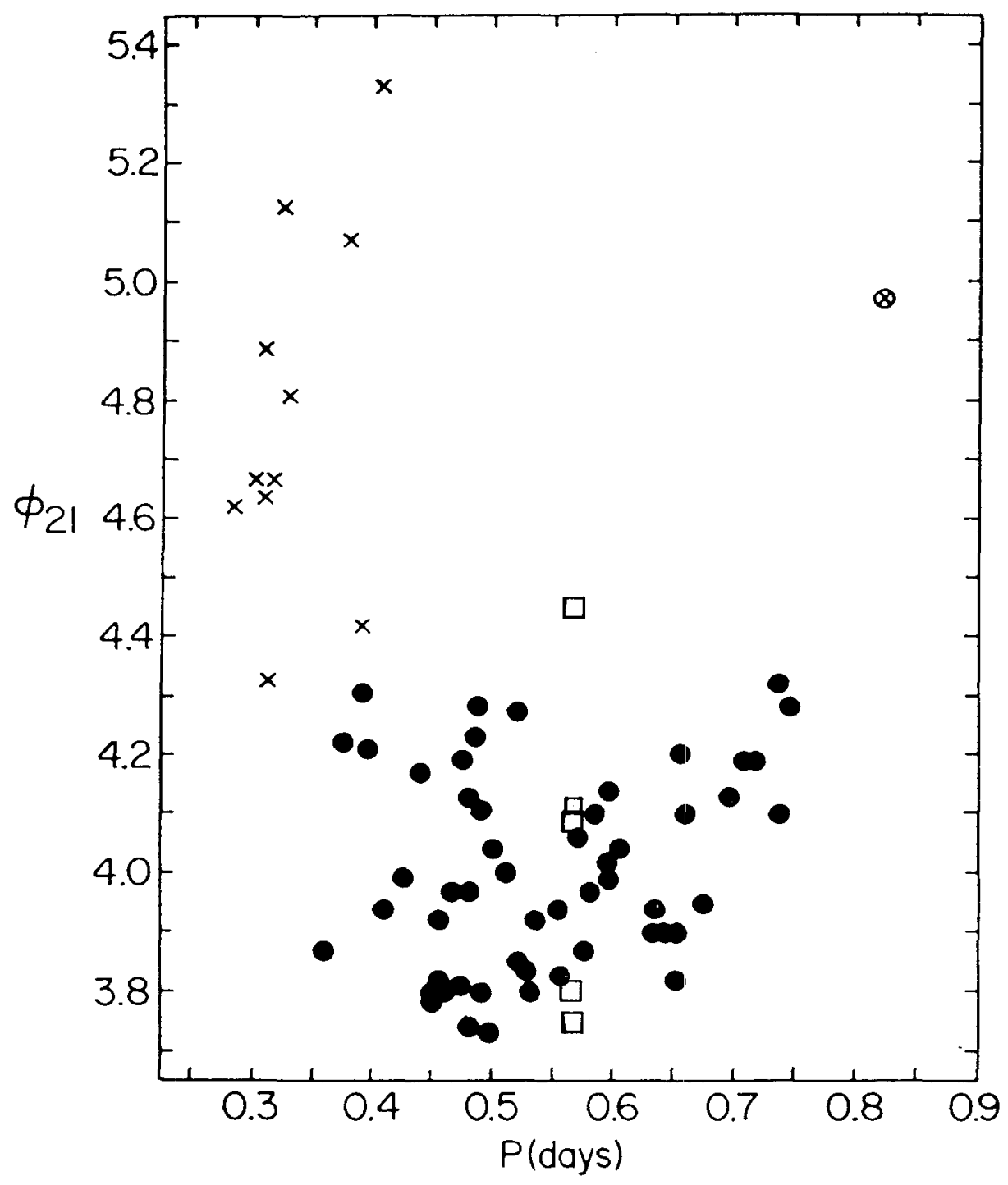

Figure 2. The Fourier decomposition parameter $\phi_{21}$ vs. period for non-Blazhko field RR Lyr stars (filled circles) and RR Lyr (open squares) at various Blazhko phases. The Fourier parameters are those defined by Simon \& Teays (1982). (Crosses represent overtone pulsators) 


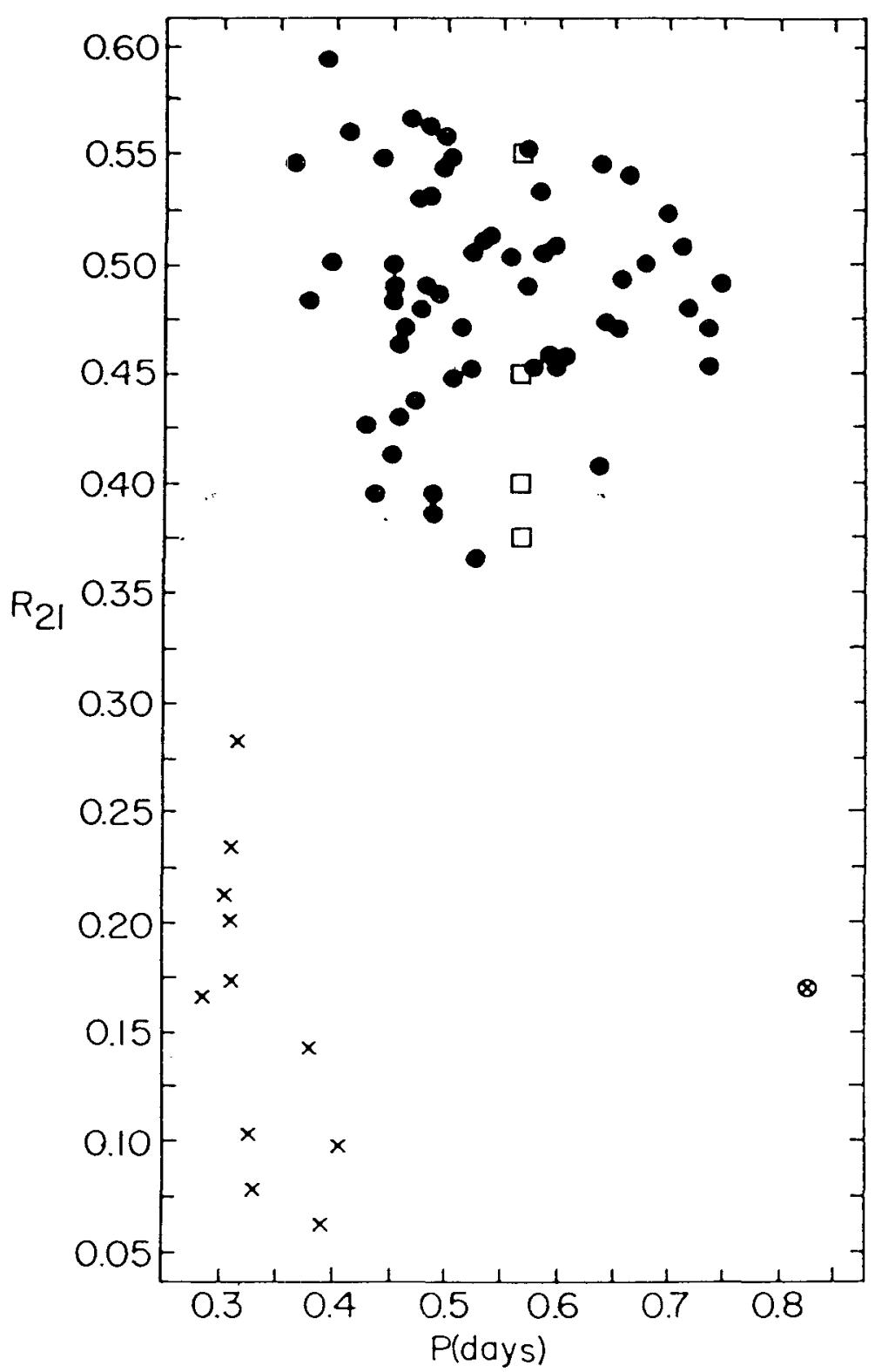

Figure 3. The Fourier decomposition parameter $R_{21}$ plotted vs. period. The symbols are the same as in Figure 2. 
at the Blazhko phase which corresponds to the largest amplitude.

Additional work is planned on these data, including seeing if one can determine phase shifts in maximum light as a function of wavelength, which can provide a useful test of pulsation models. So far, the only comparisons to models that we have performed is to the one-zone model of Grieco \& Antonello (1990), the numerical values of which were provided to us by Antonello (private communication). The predicted and observed amplitudes match rather well, including the V, LWP and SWP wavelength regions. The closest match is for the largest amplitude Blazhko phase.

\section{Concluding Remarks}

This review has summarized the characteristics that must be explained by any theory of the Blazhko effect. Our intent in conducting the observing campaigns described in $\S 3$ was to provide a body of observations which cover complete pulsation cycles at various Blazhko phases, in order to make more detailed comparisons to theory possible. Clearly there is a great deal of analysis still to be done on these data, but it is also clear that (as usual) more studies are needed. The importance of intense coordinated campaigns for the study of phenomenon like the Blazhko effect is evident.

\section{References:}

Blazhko, S. 1907, Astr. Nachr., 175, 325.

Borkowski, K. J. 1980, Space Sci. Rev., 27, 511.

Cousens, A. 1983, M.N.R.A.S., 200, 807.

Cox, A. N. 1992, (this conference).

Grieco, A. \& Antonello, E. 1990, in Confrontation between Stellar Pulsation and Evolution, ed. C. Cacciari \& G. Clementini, (San Francisco: A.S.P.), p. 101.

Moskalik, P. 1986, Acta Astron., 36, 333.

Pérez, M., Loomis, C., Eaton, N., \& Bradley, R. 1991, Report to the IUE Three-Agency Coordination Committee.

Preston, G. W., Smak, J., \& Paczyński, B. 1965, Ap. J. Suppl., 12, 98.

Romanov, Yu. S., Udovichenko, S. N., \& Frolov, M. S. 1987, Sov. Astron. Lett., 13, 29.

Simon, N. R. \& Lee, A. S. 1981, Ap. J., 248, 291.

Simon, N. R. \& Teays, T. J. 1982, Ap. J., 261, 586.

Szeidl, B., 1988, in Multimode Stellar Pulsations, eds. G. Kovács, L. Szabados, B. Szeidl (Budapest: Konkoly Observatory), p. 45.

Walraven, Th. $1949, B . A . N ., 11,17$. 


\section{DISCUSSION}

P.MOSKALIK: Is the scatter in $\phi_{21}$ vs. Period (Simon \& Teays 1982) due to Blazhko effect?

T.TEAYS: No, the stars in Simon \& Teays were carefully selected to be ones which did not show Blazhko effect.

S. SREENIVASAN: Could one not look for Zeeman splitting measurements of the magnetic field?

T. TEAYS: It is difficult, since one needs fairly high resolution, and RR Lyr stars are faint. A fall-back approach might be to compare the equivalent widths of lines which are magnetic proxies and those which are essentially unaffected by a magnetic field, as a function of phase.

G. MATHYS: About the magnetic field, one should be aware that what the Russian group measured through polarimetry in RR Lyr is the line-of-sight component of the field, which can only be detected if the field has a sufficient large-scale organization. If one determine the field from unpolarized line shape, what one gets is the field modulus, which is different. Still, it would be valuable to confirm the polarimetric detection. I believe that the magnitude limitation should not be taken seriously. Indeed, I have been measuring fields in 12th magnitude stars from spectra recorded in circular polarization at ESO. The only requirement is that the spectral lines should not be too broad.

T. TEAYS: I agree, and I urge observers to try and confirm the magnetic field measurements in RR Lyr as a function of pulsation and Blazhko phase, since a definite answer would go a long way towards determining the cause of the phenomenon.

E. ANTONELLO: I am amazed at the qualitative agreement between the model predictions and the observations in the far UV region. Since the one zone model takes into account simply the static model atmospheres, I think the shock effects, which are presumably larger in the UV region, are possible less strong than what is suspected.

T. TEAYS: It's true that the good agreement may be partly fortuitous. As I mentioned, I selected the best match to show today. Your one-zone model appears to be generally in agreement with observations from $V$ to $\approx 1500 \AA$. 